\title{
Cationic Nanoparticle-mediated Activation of Natural Killer Cells for Effective Cancer Immunotherapy
}

Kwang-Soo Kim, ${ }^{1,2,3 \dagger}$ Jun-Hyeok Han, ${ }^{4,5 \dagger}$ Seung Hee Choi, ${ }^{1 \dagger}$ Hae-Yun Jung, ${ }^{1}$ Joo Dong Park, ${ }^{1}$ Hee-Jung An, ${ }^{6}$ Seong-Eun Kim, ${ }^{7}$ Dong-Hyun Kim, ${ }^{2,3,8,9}$ Junsang Doh, ${ }^{10}$ Dong Keun Han, ${ }^{1}$ Ik-Hwan Kim, ${ }^{5}$ Wooram Park, ${ }^{4 *}$ and Kyung-Soon Park ${ }^{1 *}$

${ }^{1}$ Department of Biomedical Science, CHA University, Seongnam 13496, Republic of Korea

${ }^{2}$ Department of Radiology, Feinberg School of Medicine, Northwestern University, Chicago, Illinois 60611, United States

${ }^{3}$ Robert H. Lurie Comprehensive Cancer Center, Chicago, Illinois 60611, United States

${ }^{4}$ Department of Biomedical-Chemical Engineering, The Catholic University of Korea, Bucheon 14662, Republic of Korea

${ }^{5}$ Department of Biological Science, Korea University, Seoul 02841, Republic of Korea

${ }^{6}$ Department of Pathology, CHA Bundang Medical Center, CHA University, Seongnam 13496, Republic of Korea

${ }^{7}$ Department of Mechanical Engineering, Pohang University of Science and Technology (POSTECH), Pohang 37673, Republic of Korea

${ }^{8}$ Department of Biomedical Engineering, McCormick School of Engineering, Evanston 60208, United States

${ }^{9}$ Department of Bioengineering, University of Illinois at Chicago, Chicago 60607, United States

${ }^{10}$ Department of Materials Science and Engineering, Research Institute of Advanced Materials (RIAM), Institute of Engineering Research, Seoul National University, Seoul 08826, Republic of Korea

${ }^{\dagger}$ These authors equally contributed to this work.

*Corresponding authors

kspark@cha.ac.kr (K.-S.P.) and wrpark@catholic.ac.kr (W.P.) 

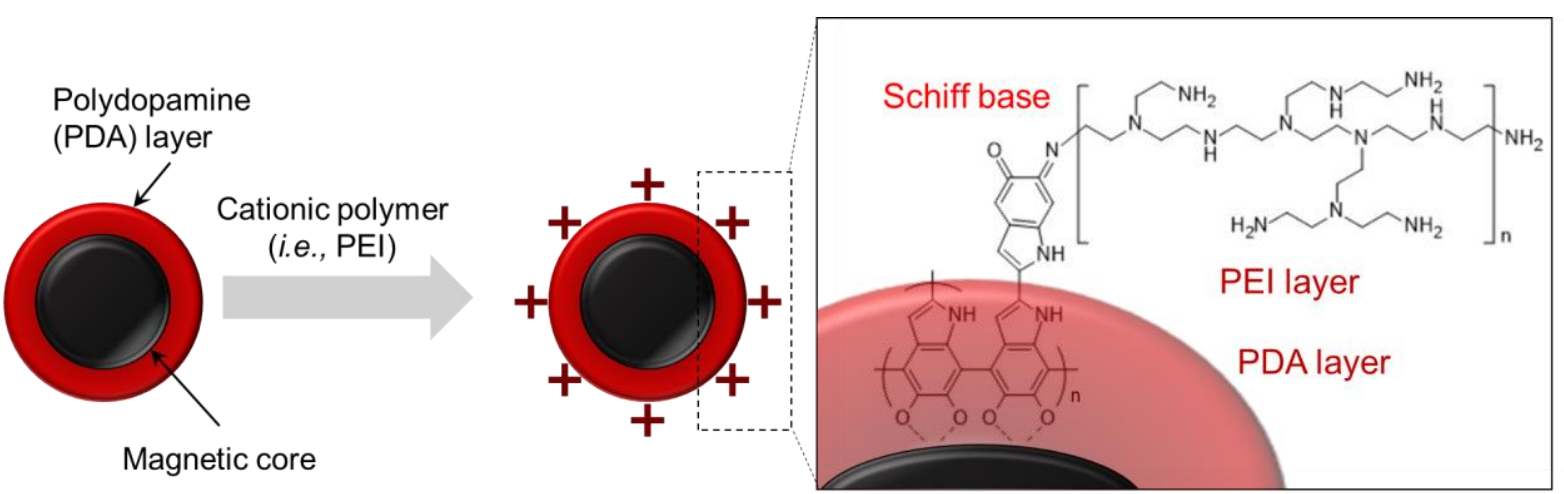

Scheme S1. Schematic illustration showing the synthesis of cationic nanoparticles (cNPs). First, the surface of magnetic nanoparticles was coated with polydopamine (PDA). The cationic polymer (i.e., polyethylenimine; PEI) was then immobilized with the PDA layer. 


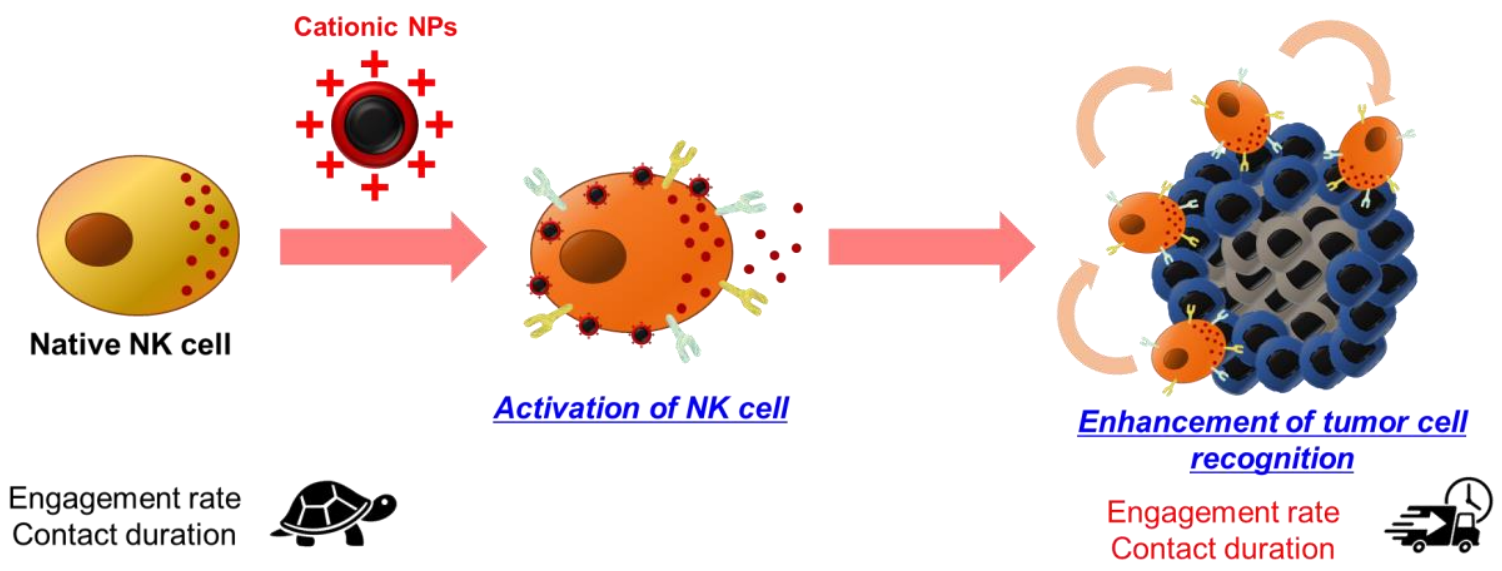

Scheme S2. Schematic illustration showed increased NK cell recognition after treatment of cationic nanoparticles (cNPs). NK cells activated by cationic nanoparticles more effectively recognize tumor cells; the polarization time is reduced time and the contact time with tumor cells increases. 
Table S1. Antibodies used for flow cytometry

\begin{tabular}{|c|c|c|}
\hline Antibody & Company & $\begin{array}{c}\text { Labeled } \\
\text { fluorescent dye }\end{array}$ \\
\hline CD11a & BioLegend & \multirow{14}{*}{$\mathrm{PE}$} \\
\hline CD16 & Invitrogen & \\
\hline CD226 & $\mathrm{BD}$ & \\
\hline CD314 & $\mathrm{BD}$ & \\
\hline CD335 & $\mathrm{BD}$ & \\
\hline CD336 & $\mathrm{BD}$ & \\
\hline CD337 & $\mathrm{BD}$ & \\
\hline $\mathrm{CD} 49 \mathrm{a}$ & BioLegend & \\
\hline CD49b & BioLegend & \\
\hline CD69 & $\mathrm{BD}$ & \\
\hline CD96 & $\mathrm{BD}$ & \\
\hline CXCR2 & eBioscience & \\
\hline Integrin beta 7 & eBioscience & \\
\hline Isotype control & $\mathrm{BD}$ & \\
\hline CCR5 & Invitrogen & \multirow{6}{*}{ FITC } \\
\hline CCR7 & $\mathrm{BD}$ & \\
\hline CD18 & $\mathrm{BD}$ & \\
\hline $\mathrm{CD} 2$ & eBioscience & \\
\hline CXCR1 & eBioscience & \\
\hline Isotype control & $\mathrm{BD}$ & \\
\hline CD193 & $\mathrm{BD}$ & \multirow{3}{*}{ PC Cy5.5 } \\
\hline CXCR3 & BioLegend & \\
\hline Isotype control & $\mathrm{BD}$ & \\
\hline CCR4 & Miltenyi & \multirow{3}{*}{ APC } \\
\hline CD107a & $\mathrm{BD}$ & \\
\hline Isotype control & $\mathrm{BD}$ & \\
\hline CCR6 & BioLegend & \multirow{3}{*}{ APC/Cy7 } \\
\hline CD62L & BioLegend & \\
\hline Isotype control & BioLegend & \\
\hline CXCR6 & R\&D system & \multirow{2}{*}{ Alexa Fluor 750} \\
\hline Isotype control & R\&D system & \\
\hline
\end{tabular}




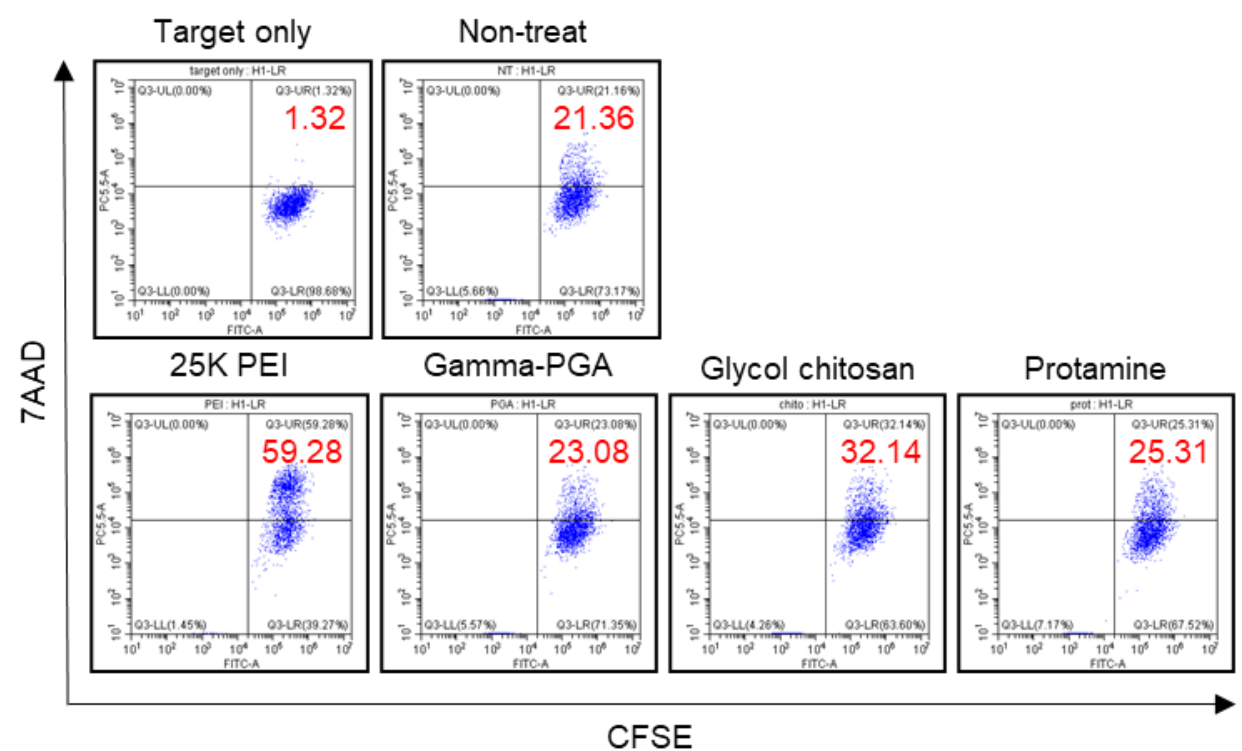

Figure S1. In vitro cytotoxic activity of NK-92MI cells treated with various cationic polymers against MDA-MB-231 was analyzed by CFSE/7AAD staining. Gamma-PGA was used as a control as an anionic polymer. Gamma-PGA did not significantly activate NK cells. 
(a)

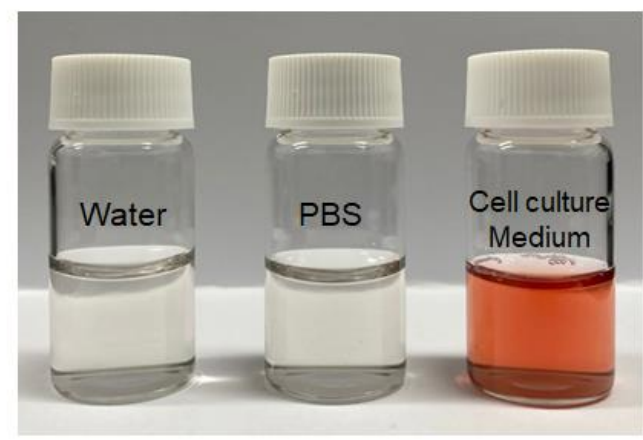

(b)

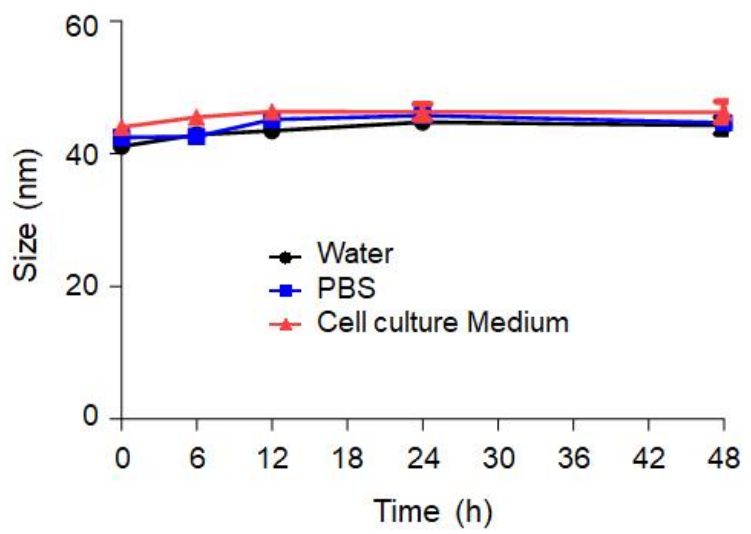

Figure S2. In vitro colloidal stability of cNPs dissolved in different dispersion medium. (a) Digital photographs of different types of dispersion medium in which cNPs are dispersed. The cNPs were dispersed at a concentration of $200 \mu \mathrm{g} / \mathrm{mL}$ in water, PBS, and cell culture medium, respectively. Digital photography was taken $48 \mathrm{~h}$ later. (b) Changes in particle size of cNPs over time in different dispersion medium. The nanoparticles were stably dispersed in all solutions, and no aggregation was observed in dynamic light scattering (DLS). 


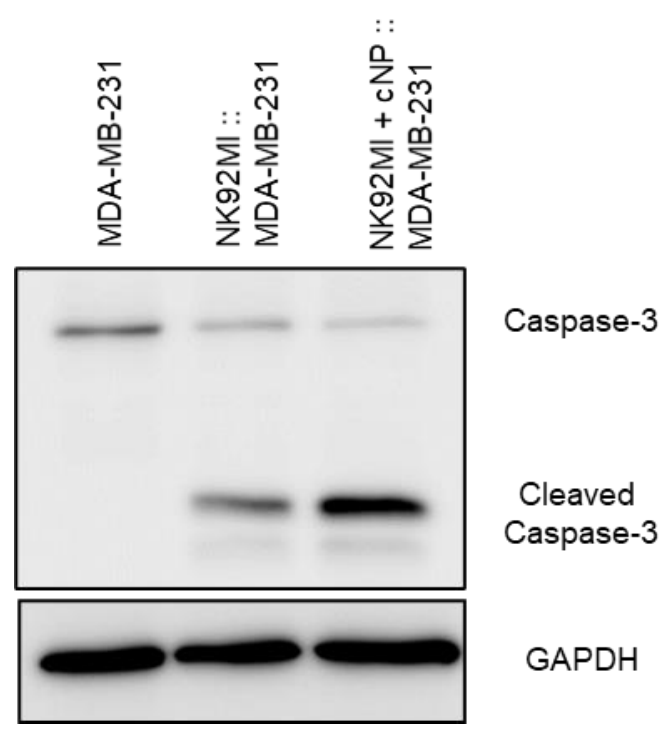

Figure S3. Immunoblot detection of cleaved caspase-3 of MDA-MB-231 cells co-incubated with NK-92MI cells treated (or not) with cNPs. NK-92MI cells were treated with cNPs for $48 \mathrm{~h}$ and then coincubated with MDA-MB-231 cells for $4 \mathrm{~h}$. Total and cleaved form of caspase3 was analyzed by immunoblot assay. 
IC

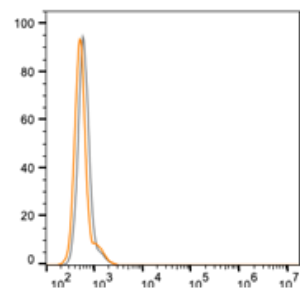

CD16

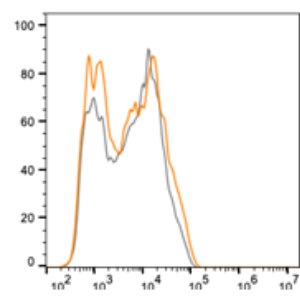

CD226

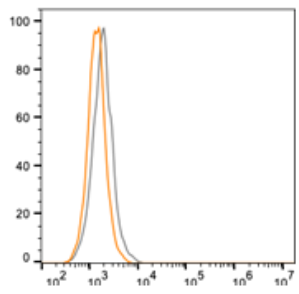

CD69

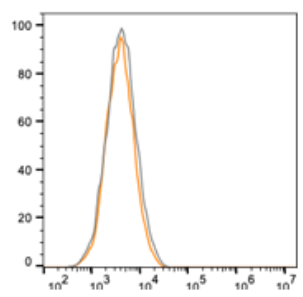

CD335

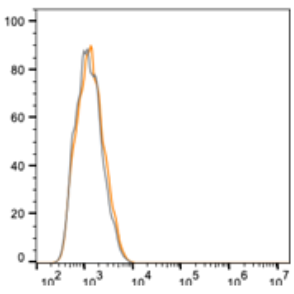

CD96

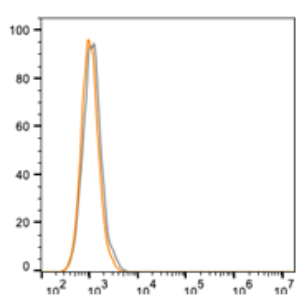

CD336

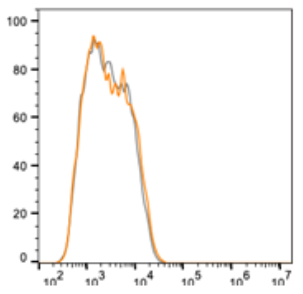

NKG2D

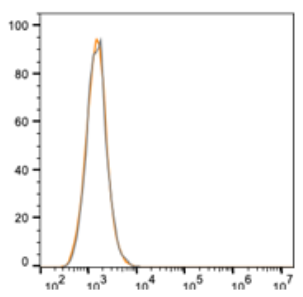

CD337

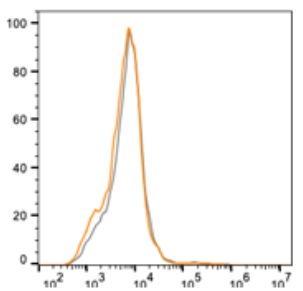

NKG2A

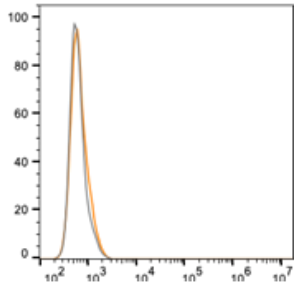

- $\mathrm{pNK}+\mathrm{DW} \quad \mathrm{pNK}+\mathrm{cNP}$

Figure S4. Changes in surface marker expression by primary NK cells (pNK) exposed toy cNPs. First, pNK cells were treated with cNPs for $48 \mathrm{~h}$. Next, expression of the indicated receptors was analyzed by flow cytometry. 
(a)

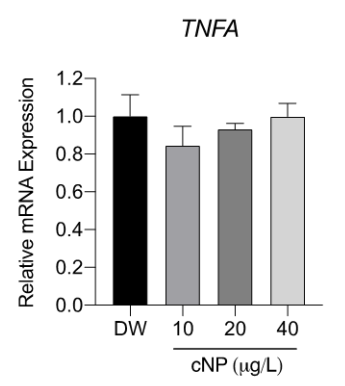

(b)

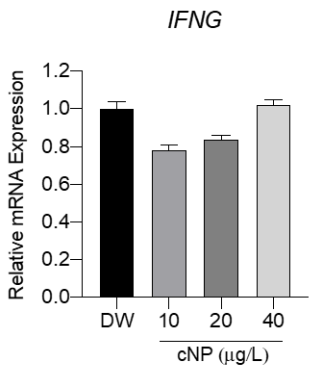

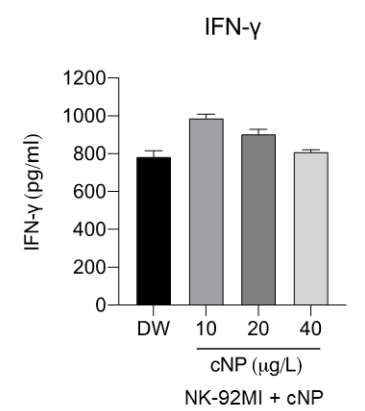

Figure S5. Quantitative analysis of TNF- $\alpha$ and IFN- $\gamma$ expression by NK-92MI cells treated with cNPs. (a) Quantitative RT-PCR analysis of mRNA encoding TNF- $\alpha$ and IFN- $\gamma$ by NK92MI cells treated for $48 \mathrm{~h}$ with the indicated concentrations of cNPs. (b) ELISA of IFN- $\gamma$ of NK-92MI cells treated for $48 \mathrm{~h}$ with the indicated concentrations of cNPs. cNP-treated NK92MI cells were incubated without (left) or with (right) MDA-MB-231 cells for $4 \mathrm{~h}$. Secretion of IFN- $\gamma$ into the culture medium was then measured. 


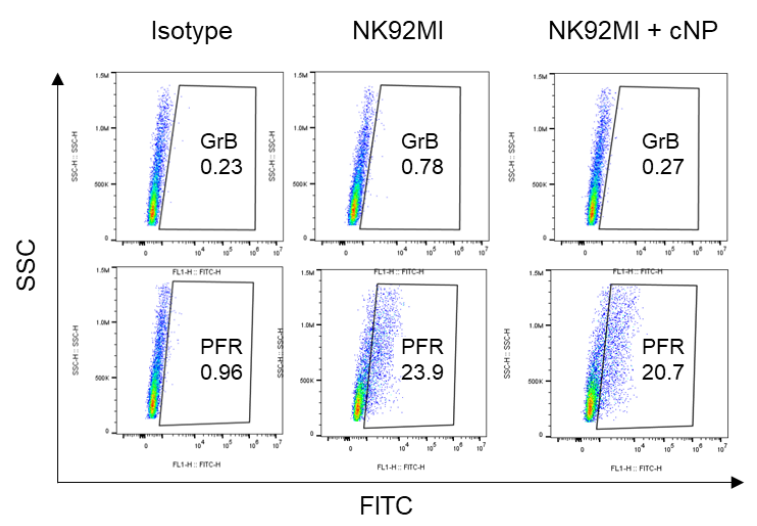

Figure S6. Quantitative flow cytometry analysis of intra-cellular granzyme B and perforin level in cNP-treated NK-92MI cells. NK-92MI cells were treated with cNPs for 48 $\mathrm{h}$ and then cocultured with MDA-MB-231 cells for $4 \mathrm{~h}$. 


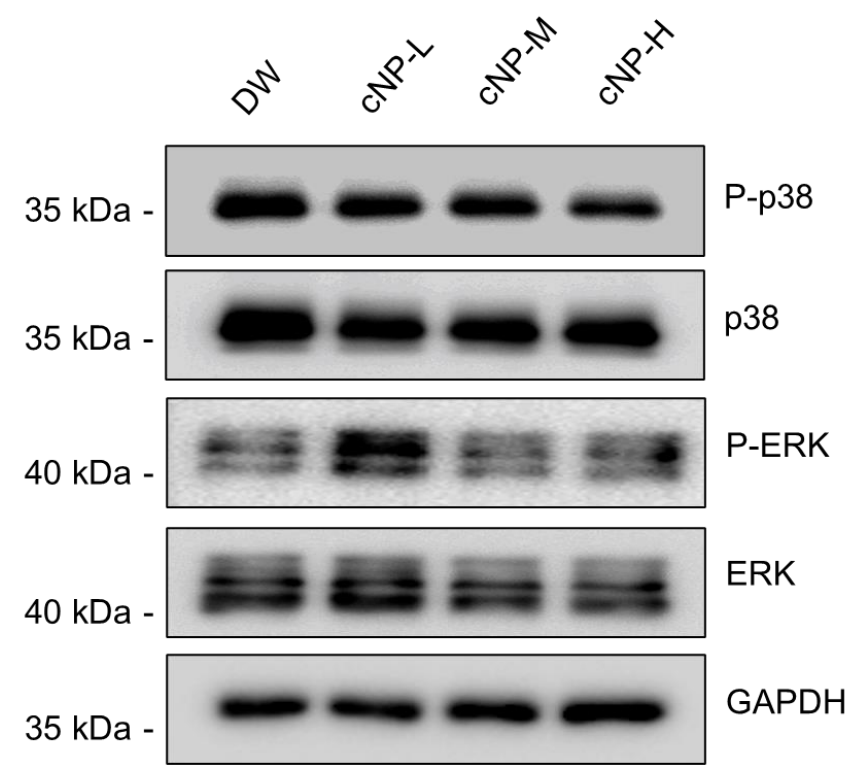

Figure S7. Effect of cNPs on activation of signaling pathways related to NK cell activity. NK-92MI cells were treated for $48 \mathrm{~h}$ with the indicated concentrations of cNP and total protein levels were analyzed by immunoblotting with the indicated antibodies. 
(a)

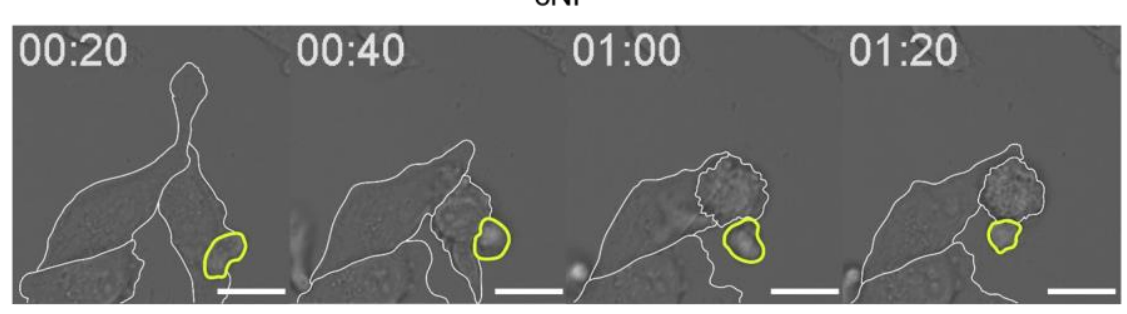

(b)

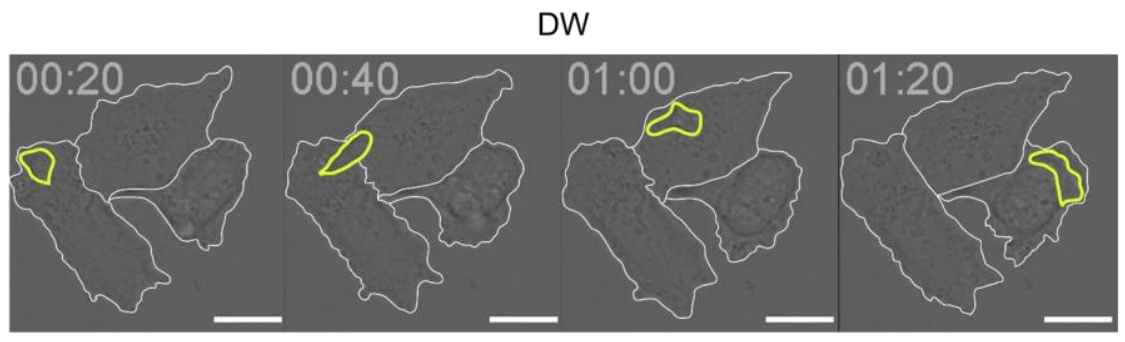

(c)

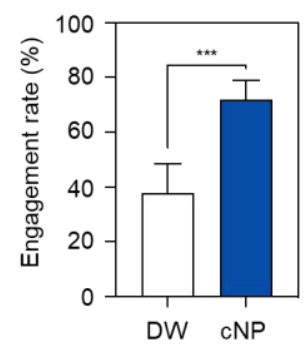

(d)

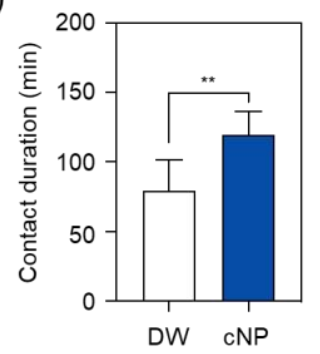

Figure S8. Direct visualization of the primary NK-MDA-MB231 interactions using timelapse images. ( $a, b)$ Representative time-lapse images of cNP-treated (a) or control (b) pNK cells interacting with cancer cells. Elapsed time: hh:mm. Scale bars: $10 \mu \mathrm{m}$ (c) Engagement rate of pNK cells encountering MDA-MB231 cells. (d) Contact duration of pNK cells interacting stably with MDA-MB231 cells. 

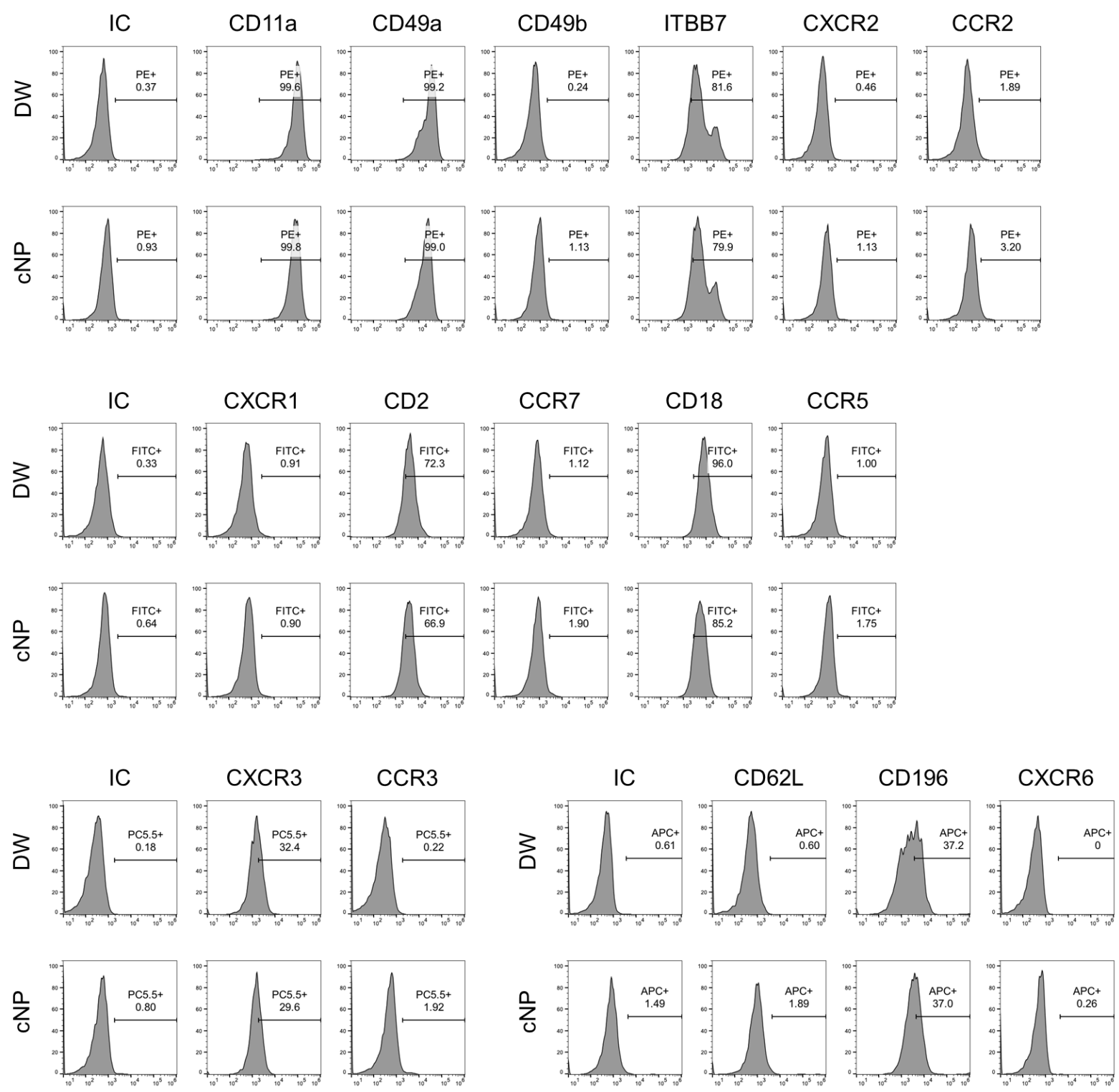

Figure S9. Changes in adhesion and chemokine receptor expression by NK-92MI cells exposed to cNPs. NK-92MI cells were treated for $48 \mathrm{~h}$ with cNPs and expression of the indicated receptors was analyzed by flow cytometry. 

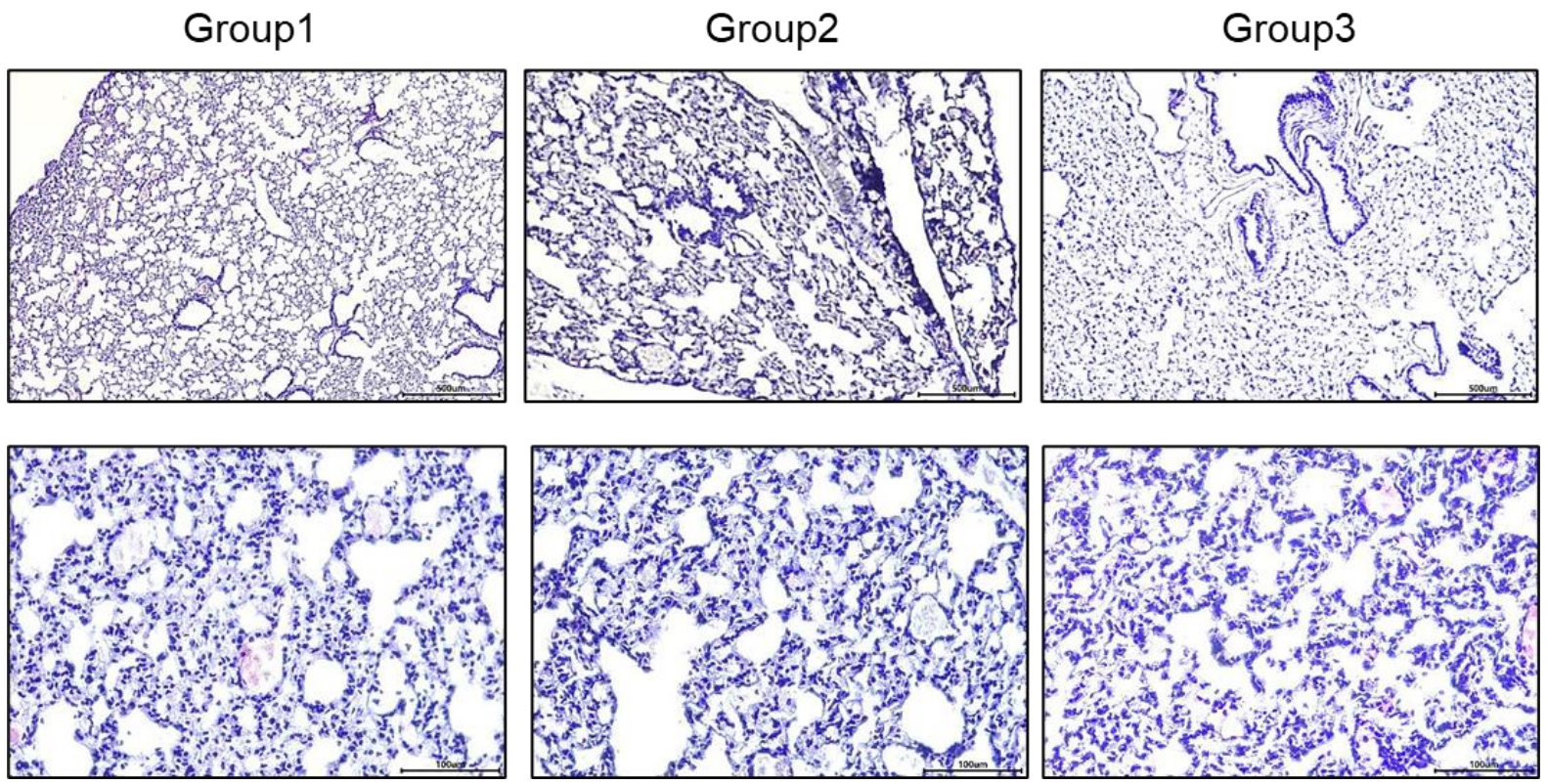

Figure S10. Effect of cNP-treated (or not) NK-92MI injection on the lung of the tumor xenograft mouse model (Group 1, treated with DPBS; Group 2, treated with NK-92MI; Group 3, treated with cNP-treated NK-92MI). Lung sections of each group were analyzed by H\&E staining. 

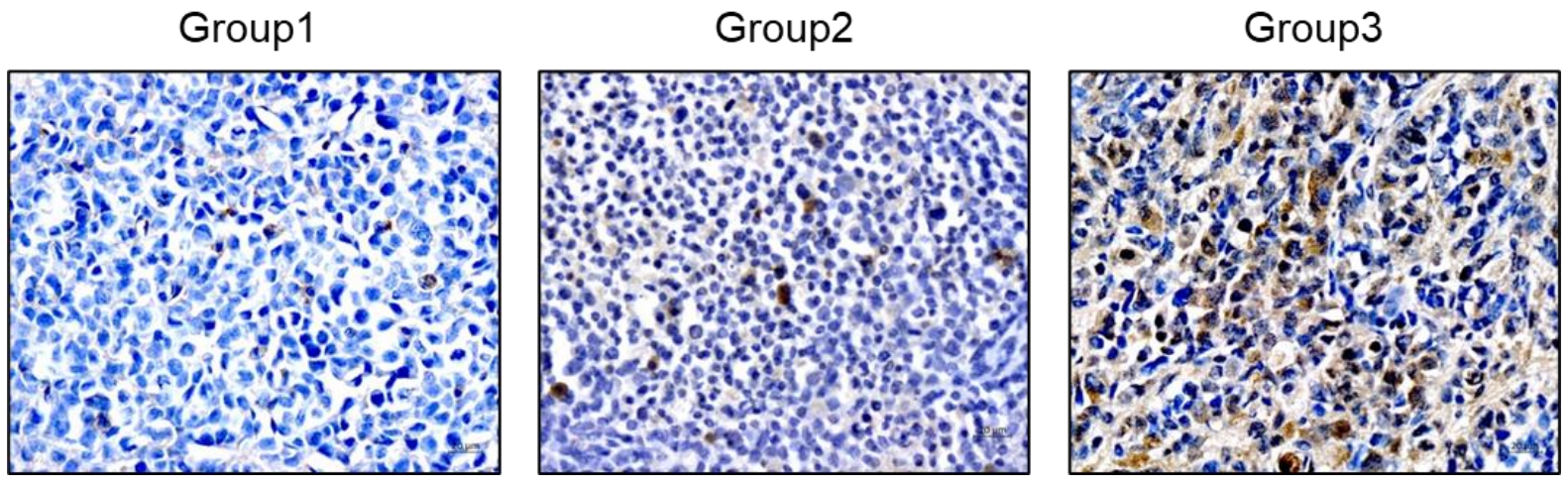

Figure S11. Immunohistochemical staining of cleaved caspase-3 with the tumor sections of MDA-MB-231 xenograft mouse model (Group 1, treated with DPBS; Group 2, treated with NK-92MI; Group 3, treated with cNP-treated NK-92MI). 
(a)

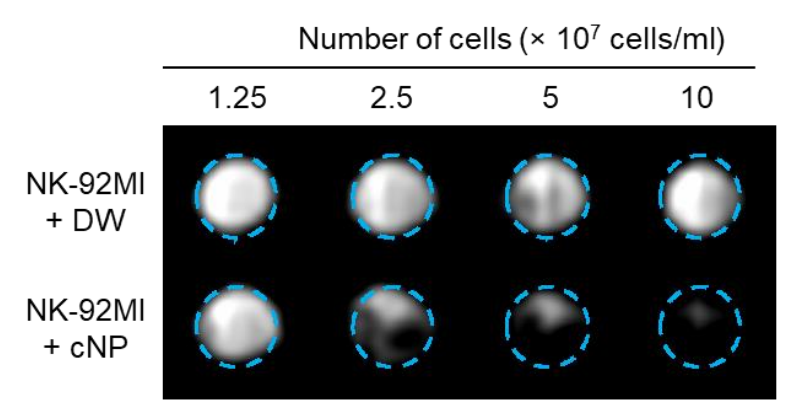

(b)

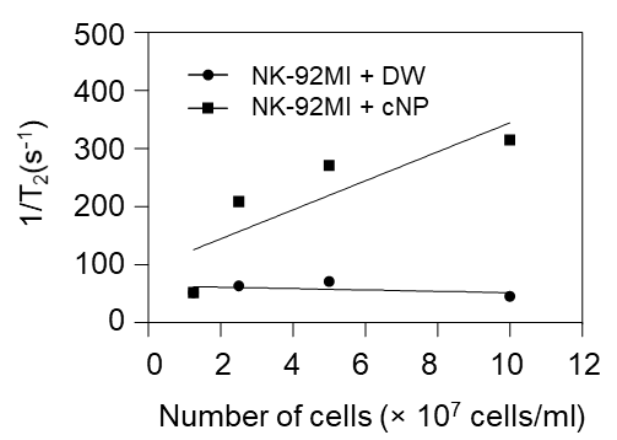

Figure S12. Magnetic resonance (MR) imaging of NK-92MI cells treated with cNPs. (a) $T_{2}$-weighted MR images showing NK-92MI cells treated with cNPs in $1 \%$ agar phantoms. (b) $1 / T_{2}$ plot of the number of NK-92MI cells after exposure to cNPs. 